\title{
Neurocysticercosis in an Oncology Patient with High Risk Stage 1 Endometrial Cancer
}

\section{Price $\mathrm{L}^{*}$ and Allerton $\mathrm{R}$}

The Deansley Centre, Royal Wolverhampton Hospital, Wolverhampton, UK

*Corresponding author: Price L, The Deansley Centre, Royal Wolverhampton Hospital, Wolverhampton, UK, Tel: +441902307999; E-mail: louise.price12@nhs.net Received Date: Sep 06, 2018; Accepted Date: Sep 14, 2018; Published Date: Sep 22, 2018

Copyright: (c) 2018 Price L, et al. This is an open-access article distributed under the terms of the Creative Commons Attribution License, which permits unrestricted use, distribution, and reproduction in any medium, provided the original author and source are credited.

\begin{abstract}
Our 53-year-old patient underwent Total Abdominal Hysterectomy (TAH) and Bilateral Salpingo-Oopherectomy (BSO) for Stage 1B, mixed serous and endometroid adenocarcinoma. Post operatively she had no residual disease. Otherwise she had longstanding Rheumatoid arthritis for which she received Methotrexate 3 times per week. She received post-operative adjuvant single agent Carboplatin chemotherapy. Treatment was limited to 4 cycles due to myelosuppression, which was confounded by the previous use of Methotrexate. It was planned to undergo internal vaginal vault Brachytherapy. Less than 6 months following TAH and BSO she presented with sudden onset expressive dysphasia. CT and MRI imaging of the brain demonstrated an irregular enhancing lesion in the left temporal lobe with vasogenic cerebral oedema. The clinical concern was that she had developed cerebral metastasis. She was treated acutely with Dexamethasone $8 \mathrm{mg}$ BD, craniotomy and excision and pathological analysis of the lesion confirmed Cysticercosis. This novel case reminds us that biopsy of new lesion in a patient with cancer is vital.
\end{abstract}

Keywords: Neurocysticercosis; Oncology patient; Endometrial cancer

\section{Background}

This case describes a 53-year-old with locally excised stage 1B (FIGO, 2009) mixed serous and endometroid adenocarcinoma. Therefore her disease was high risk endometrial cancer [1] and she went on to have chemotherapy. Within 12 months she presented acutely with a cerebral lesion. For intermediate and high risk, early stage endometrial cancer rates of distant metastases are quoted by PORTEC2 as between 5.7-8.3\% for those treated with local radiotherapy only [2].

Our patient had a single lesion and it is estimated that $30-40 \%$ of patients with cerebral metastases present with a single lesion and the remainder with multiple lesions [3]. The management includes the use of steroids to control pressure symptoms, plus either best supportive care or active treatment which includes either whole brain radiotherapy, Steriotactic radiotherapy (SRS) and Surgical resection of cerebral metastases or a combination of active treatments.

For consideration of SRS patients must be discussed at the local Neurology multidisciplinary team (MDT). From there if deemed appropriate they are then referred to SRS centers for MDT discussion with both specialist neurosurgery and specialist clinical oncology teams.

Criteria for SRS includes Karnofsky performance status (KPS) more than 70, life expectancy greater than 6 months, an established diagnosis of cancer, controlled primary disease, no neurological symptoms of raised intracranial pressure or features best relieved by surgery, tumour volume of less than $20 \mathrm{cc}$ on pre-treatment scans [4]. Our patient met these criteria for SRS to be considered. Due to the positioning of the lesion, the consensus was to perform craniotomy and de-bulking for diagnostic and treatment purposes.
Histology revealed Cysticercosis. The eggs of the tapeworm (Taenia solium) are ingested via the fecal-oral route and as they reach the stomach are partially degraded by gastric acid into larval cysts. The oncospheres form cysts in the wall of the intestine. From there they can enter the vascular system and then form cysts within the muscles, brain and eye. When the cysts invade the nervous system this is called Neurocysticercosis [5].

Cysticercosis is rare in the UK but incidence is increasing in the western world, thus the importance of this case [6]. Neurocysticercosis is the most common cause of epilepsy worldwide, with the endemic areas within the developing world. However, with global travel, the incidence in the USA has increased [6].

\section{Case Report}

This unique case describes a well 53-year-old lady who initially presented Menorrhagia and a raised CA-125 of $400 \mathrm{KU} / \mathrm{l}$. She had routine Total Abdominal Hysterectomy and Bilateral SalpingoOopherectomy, from which histology demonstrated a mixed Serous and Endometroid Adenocarcinoma, involving more than half of the myometrium with no lymphovascular invasion and a CT chest, abdomen and pelvis showed no distant disease (FIGO Stage 1b) [7].

She has a past medical history of Rheumatoid arthritis treated with 3 times a week Methotrexate. In addition, she takes Folic acid and as an analgesic Solpadol together with Celecoxib and Lansoprazole. Otherwise she is well, KPS of 0 , and has never smoked, she takes less than $21 \mathrm{IU}$ of alcohol and works as a Tax manager. She has no history of BRACA associated tumours but her mother died of Lung cancer.

Due to the poorly differentiated serous component of the histological specimen she was offered platinum based chemotherapy (per EORTC-55991 and MaNGO ILIADE-III trial) [8]. 
The option of Carboplatin Paclitaxel was discussed together with the associated toxicities. She had a theoretical increased risk of myelosuppression due to previous treatment with Methotrexate. In collaborative discussion with the patient she opted for single Carboplatin chemotherapy (AUC 6 every 21 days for 6 cycles). However single agent Carboplatin chemotherapy was discontinued after completion of 4 cycles due to myelosuppression.

Standard treatment of vaginal vault brachytherapy $(22 \mathrm{~Gy}$ at $0.5 \mathrm{~cm}$ for $2 \mathrm{~cm}$ vaginal length over 2 weeks) to reduce the risk of local recurrence was planned. However this did not occur due to the patient being a virgin and the emotional turmoil associated with the treatment.

4 months later she presented as an emergency with acute expressive dysphasia and problems reading and writing. She had no seizure activity or further neurological symptoms. She had no gastrointestinal symptoms or masses and her Eosinophils were normal.

CT head imaging was carried out and demonstrated a corresponding less than $30 \mathrm{~mm}$ in diameter $(19 \times 19 \times 14 \mathrm{~mm})$ of the left temporal lobe with an enhancing rim. MRI T2 FLAIR. Feb 2017. Demonstrating left posterior temporal lesion with ring enhancement with central non-enhancement (in keeping with central necrosis) (Figure 1).

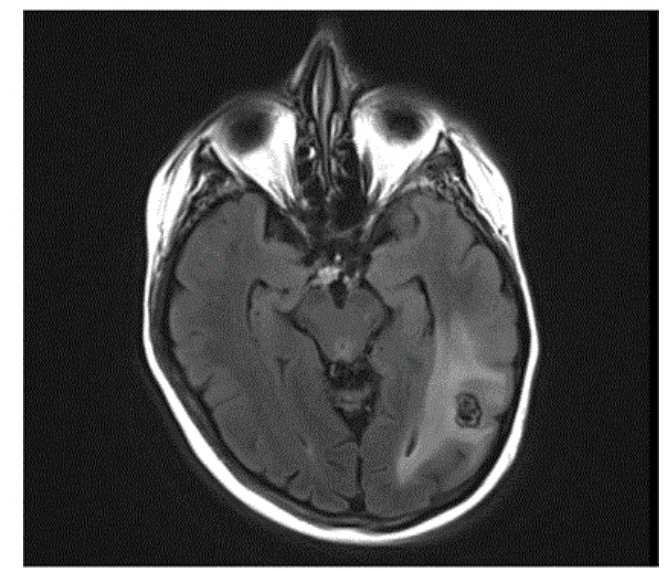

Figure 1: MRI T2 FLAIR.

She was started on steroids with some symptomatic improvement. The initial clinical concern was that this was a metastatic deposit. The lesion was further characterised by an MRI brain plus contrast which was not characteristic of a metastatic deposit. She underwent whole body CT staging of the chest, abdomen and pelvis that ruled out further sites of disease. She subsequently underwent Neurosurgical excision of the lesion to which the histology was surprising.

The histology demonstrated Cysticercosis (Figure 2). With hindsight, she had travel history to endemic areas of South America, Africa and Asia [9]. As within the last two years has travelled to Mexico, Guatemala, Ecuador, Cube, Egypt, Namibia, Jordan, Oman, India, Burma, Vietnam, Cambodia and China. The patient had no muscular masses on examination and no gastrointestinal symptoms of note; clinically this was an isolated Cysticercosis cyst. Had the case been treated as distant metastasis from endometrial cancer with radiotherapy to the cerebral lesion the diagnosis would have been missed, the patient would have been given incorrect prognostic information and given therapy with significant morbidity with a risk of mortality. Other less likely differentials include bacterial, viral infections, infarction, hemorrhagic and inflammatory conditions.

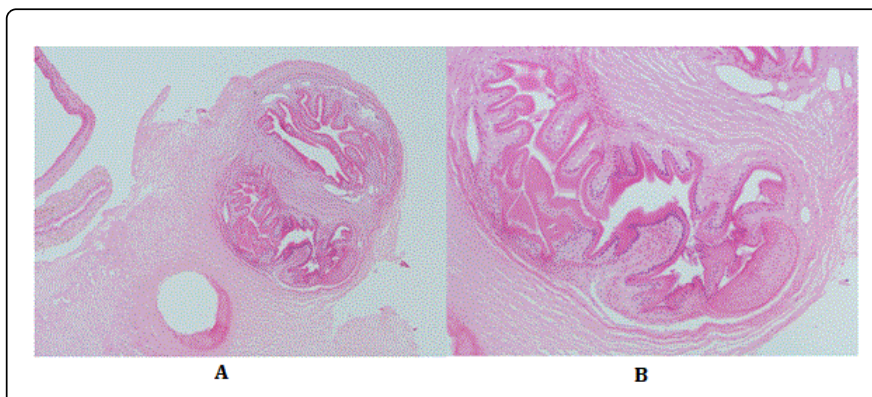

Figure 2: Histology showing (A) the cystic cavity and (B) granulomatous reaction, inflammatory infiltrate with lymphocytes and eosinophils, fibrosis and calcification.

Following recovery from her Craniotomy and excision she was treated for Cysticercosis with the anti-parasitic Albendazole $400 \mathrm{mg}$ twice a day plus dexamethasone. The rationale was that she had had prolonged immunosuppression with Methotrexate and then later Carboplatin so after discussion treatment was given, although this approach can be debated [6].

Two years following initial presentation and subsequent treatment for incidental Cysticercosis the patient remains well and works full time and continues to enjoy travelling!

\section{Discussion}

Our patient had tumour invasion of more than $50 \%$ of the myometrium, FIGO 2009 Stage 1b [7] demonstrating mixed adenocarcinoma and serous histology without lymphovascular invasion.

The PORTEC1 trial initially stratified FIGO stage I endometrial tumours into low, intermediate and high risk by considering; Histological subtype, grade, presence of lymphovascular invasion, patient age and number of lymph nodes sampled [10]. Our patient is high risk due to high grade histology with a serous component.

Our patient was due to receive post-operative CarboplatinPaclitaxol chemotherapy followed by vaginal vault brachytherapy. The evidence for which we shall outline.

Currently the role of adjuvant chemotherapy and concurrent chemoradiotherapy in high risk Stage I endometrial cancer patients is being addressed by the Phase III multicenter international study PORTEC-3 which has yet to publish mature data [11]. In 2017, the practice of giving adjuvant chemotherapy is evidenced by combined analysis of the EORTC-55991 and MaNGO ILIADE-III trials showing a $36 \%$ reduction in relapse in high risk endometrial cancer patients without post-operative residual disease [8]. It has been shown for patients like ours, with serous histology treatment with a cisplatin based regime is favorable [12].

PORTEC1 concluded post-operative adjuvant external beam radiotherapy (EBRT) is beneficial, reporting 5 year local recurrence rates of $4 \%$ versus $14 \%$ in Radiotherapy and control groups respectively $\mathrm{p}<0.001)$ [10]. Later PORTEC2 demonstrated vaginal 
brachytherapy and EBRT treatments are equivocal in local control rates with vaginal vault brachytherapy giving bowel toxicity $[1,2]$.

Our patient developed a cerebral lesion before embarking on vaginal vault brachytherapy, so was investigated acutely with CT and MRI brain scans plus CT chest, abdomen and pelvis. The results of which were discussed at the tertiary MDT meeting, involving neurosurgeons, neuro-radiologists, oncologists, neurologists and pathologists. The decision was to surgically remove it due to the position and discrete nature of the lesion in a patient with a good performance status. The lesion was not metastatic disease but Cysticercosis. Had the case been treated as distant metastasis from endometrial cancer with radiotherapy to the cerebral lesion the diagnosis would have been missed, the patient would have been given incorrect prognostic information.

Cysticercosis develops by initial ingestion of Taenia eggs via the fecal-oral route. Two months after initial ingestion of Taenia eggs nonspecific gastrointestinal symptoms develop such as abdominal bloating, nausea, constipation and diarrhea [9].

Taenia solium cysts develop within the intestine which with the rich vascular supply can form Cysticercosis cysts within the muscle, eyes and brain. Cysts within the brain can cause seizures and local disruption of neurological functioning. Our patient developed expressive dysphasia and problems reading and writing over a period of days. This corresponds with disruption of functioning in the temporal lobe.

To our knowledge there are no case reports of neurocysticercosis in patients receiving immunosuppressive chemotherapy for endometrial cancer. This case provides a reminder of the implications of prolonged immunosuppression for Rheumatological and Oncological purposes alike.

\section{Conflicts of Interest}

There is no conflict of interest or financial influence.

\section{References}

1. Kupets R, Le T (2013) The Role of Adjuvant Therapy in Endometrial Cancer. J Obstet Gynaecol Can 35: 375-376.

2. Nout RA, Smit VT, Putter H, Jürgenliemk-Schulz IM, Jobsen JJ, et al. (2010) Vaginal brachytherapy versus pelvic external beam radiotherapy for patients with endometrial cancer of high-intermediate risk (PORTEC-2): an open-label, non-inferiority, randomised trial. Lancet 375: 816-823.

3. Lohr F, Pirzkall A, Hof H, Fleckenstein K, Debus J (2001) Adjuvant treatment of brain metastases. Semin Surg Oncol 20: 50-56.

4. NHS Commissioning Board (2013) Clinical Commissioning Policy: Stereotactic Radiosurgery / Radiotherapy for Cerebral Metastases.

5. DeGiorgio CM, Medina MT, Duron R, Zee C (2004) Neurocysticercosis. Epilepsy Curr 4: 107-111.

6. Otte WM, Singla M, Sander JM, Singh G (2013) Drug therapy for solitary cysticercus granuloma: a systematic review and meta-analysis. Neurology 80: $152-162$.

7. Colombo N, Preti E, Landoni F, Carinelli S, Colombo A, et al. (2013) Endometrial cancer: ESMO Clinical Practice Guidelines for diagnosis, treatment and follow-up. Ann Oncol 24: 33-38.

8. Hogberg T, Singorelli M, Freire de Oliveira C, Fossati R, Lissoni AA, et al. (2010) Sequential adjuvant chemotherapy and radiotherapy in endometrial cancer - Results from two randomised studies. Eur J Cancer 13: $2422-2431$.

9. http://www.who.int/mediacentre/factsheets/fs376/en/

10. Creutzberg CL, van Putten WL, Koper PC, Lybeert ML, Jobsen JJ, et al. (2000) Surgery and postoperative radiotherapy versus surgery alone for patients with stage-1 endometrial carcinoma: multicentre randomised trial. PORTEC Study Group. Post-Operative Radiation Therapy in Endometrial Carcinoma. Lancet 355: 1404-1411.

11. de Boer SM, Powell ME, Mileshkin L, Katsaros D, Bessette P, et al. (2013) Toxicity and quality of life after adjuvant chemoradiotherapy versus radiotherapy alone for women with high-risk endometrial cancer (PORTEC-3): an open-label, multicentre, randomised, phase 3 trial. Lancet Oncol 17: 1114-1126.

12. Fader N, Drake RD, O'Malley DM, Gibbons HE, Huh WK, et al. (2009) Platinum/taxane-based chemotherapy with or without radiation therapy favorably impacts survival outcomes in stage I uterine papillary serous carcinoma. Cancer 115: 2119-2127. 